Proceedings of the 2009 Winter Simulation Conference

M. D. Rossetti, R. R. Hill, B. Johansson, A. Dunkin, and R. G. Ingalls, eds.

\title{
Duopoly Electricity Markets with Accurate and Inaccurate Market Goals
}

\author{
Zhi Zhou \\ Wai Kin Victor Chan \\ Department of Decision Sciences \& Engineering Systems \\ Rensselaer Polytechnic Institute \\ Joe H. Chow \\ Troy, NY 12180, USA \\ Department of Electrical, Computer \& Systems Engineering \\ Rensselaer Polytechnic Institute \\ Troy, NY 12180, U.S.A.
}

\author{
Serhiy Kotsan \\ New York Independent System Operator \\ Rensselaer, NY 12144
}

\begin{abstract}
Electricity markets are complex systems due to their deregulation and restructuring. We develop an agent-based simulation model for a stylized electricity pool market and simulate the market as a repeated game. An online hill climbing with adjustment algorithm is applied to generator agents to guide them to bid strategically to reach their expected market share. It is observed that accurate (or genial) expected market goals lead to collusive behavior of generator agents with an equilibrium where their total profit is maximized. On the other hand, it is also found that inaccurate (or malicious) market goals could result in price war with an equilibrium where their profits are minimized.
\end{abstract}

\section{INTRODUCTION}

An electricity market is a complex system because of the nature of the traded goods (electricity) and the complexity of the market structure and composition. It is not economic to store electricity, and hence, supply and demand must be balanced in real time subject to transmission and capacity constraints. Moreover, an electricity market itself is a combination of many submarkets, such as the day-ahead market, real-time market, bilateral market, installed capacity market, and emission market. In addition, an electricity market might also connect to other markets, such as the natural gas and petroleum market. Due to high capital investments, participants (companies) in an electricity market are relatively concentrated, resulting in the following consequences: (1) some companies may be organized vertically and participate in more than one of the submarkets simultaneously, and (2) some of the companies may be big enough to apply their market power to manipulate market prices.

Auction is a widely used mechanism to create competitive markets in homogeneous commodity exchanges such as agricultural products, mineral resources, and financial market exchange such as stocks, bonds, and treasury bills. Auction can produce efficient allocations and prices even with a rather small number of players who have incomplete or imperfect information on supply and demand (Friedman and Rust 1993). In electricity markets, auction is one of the most important mechanisms to allocate electricity, installed capacity, and transmission rights. Bidders in these markets are asymmetric and do not know the information of their opponents. However, they participate regularly to bid on the same commodity with varying quantities. Bidders with generation capacity or cost advantages may try to exercise their market power to maximize their profits. As a result, auctions in electricity markets are a repeated and asymmetric game with imperfect competition. Most existing research focuses on theoretical modeling and analysis with assumptions such as symmetric bidders, single commodity, or competitive bidding (Bower and Bunn 2001).

This paper employs an agent-based simulation (ABS) and optimization approach to analyze bidding behavior in electricity markets. We create an agent-based simulation model that mimics the evolutionary dynamics of bidding behavior in a repeated and multi-unit auction under the settings of an electricity market. The market participants are modeled as intelligent agents with non-homogenous optimization objectives and learning abilities. Our goal is to understand how market participants behave in this repeated game and what the consequences are. 


\section{Zhou, Chan, Chow and Kotsan}

The rest of the paper is organized as follows. Section 2 reviews the background and related work of electricity markets and agent-based simulations. Section 3 presents an agent-based simulation model and states the assumptions and settings of the experiments. Section 4 discusses the experimental results and Section 5 offers some conclusions.

\section{BACKGROUND}

During the last twenty years, a process has been undergone worldwide to restructure electrical power facilities and liberalize the markets for the services based on these facilities. This process moves the electricity industry from vertically integrated monopolies to more efficient, separated companies, and replaces the regulated, cost-based market to a supply and demand based competitive one. The major goal of this reform is to promote energy conservation and alternative energy technologies, and to reduce oil and gas consumption through technology improvement and regulations (FERC 2006).

Typically, two types of instruments for trading electricity are used: pool and bilateral contracts. In a pool contract market, generation and customer companies submit their bids and the market is cleared by a market operator (e.g., the Independent System Operator (ISO)). The operator also announces the clearing prices for the next day based on the amount of the supply and demand bids submitted by the producers and buyers. Because of its main usage in day-ahead and real time bidding, the pool market is considered as a short-term trading market. On the other hand, in a bilateral contract market, bilateral contracts are used by companies to hedge against the risk of daily price volatility. Bilateral markets are forward markets. The contract periods can cover six months or more.

The complexity of electricity markets has attracted attentions from many research groups since 1990. Most of the research focuses on market efficiency and generation competition. The research problems can be studied by analyzing market equilibria under different market scenarios or structures. Equilibrium theory and game theory are therefore good candidate tools for studying these types of problems.

Green and Newbery (1992) introduce a supply-function equilibrium model (Klemperer and Meyer 1989) and formulate the England and Wales electricity market as a single-shot game consisting of two symmetrical players. By finding the Nash equilibrium for the supply schedule, the authors show that a high premium on marginal costs is possible despite having a competitive market. Similarly, Rudkevich et al. (1998) estimate the price of an electricity pool market by using a closed-form mathematical formula based on the supply function equilibrium (SFE). Models based on the equilibrium theory usually assume either perfect and complete information about opponents or no strategic bidding behavior.

Another line of studies is based on game theory. von der Fehr and Harbord (1993) model the England and Wales electricity market as a first-price, sealed-bid, multi-unit, and private-valued auction market. Their results show that the bidding behavior tend to support the theoretical model. The authors claim that pure-strategy equilibria do not exist for a wide range of demand distributions. Borenstein and Bushnell (1999) consider the California electricity market after deregulation as a static Cournot market and simulate the market using historical cost data. They show that potential market power during high demand hours is possible. Game theory is a good alternative to analytically study of the market when the number of participants is small. However, with the deregulation of electricity markets, electric power systems have become more complex and involved a large number of competing participants.

Agent-based simulation (ABS) is a relatively new technique that may overcome the shortcomings of traditional methodologies. The participants are modeled as agents. Unlike traditional methodologies, ABS does not require the knowledge of perfect or complete information of participant agents. Agents are equipped with learning algorithms that could guide them to achieve their goals. ABS is a powerful tool for simulating dynamics of multi-participant systems and analyzing emerging behavior.

Bunn and Oliveira (2001) model the England and Wales electricity market under the New Electricity Trading Arrangements (NETA) using ABS. They show that electricity prices are high when the demand is in the highest range. They also observe that power plants with flexible technologies are relatively more valuable under the NETA. Nicolaisen et al. (2001) use ABS to examine market power and efficiency as functions of market concentration and capacity. The market is modeled as a double-auction market, which is cleared by a discriminatory midpoint pricing mechanism. Wu and Guo (2004) apply a reinforcement learning method-the learning automata-to the Western Systems Coordinating Council (WSCC) 9-bus test system. Micola et al. (2008) study whether a vertically integrated firm can increase its profits in oligopolistic energy markets by using interdependent rewards obtained from strategic business units. Tellidou and Bakirtzis (2007) examine the effect of participants' behavior such as capacity withholding and tacit collusion to market performance. They model the electricity market as an hourly repeated game on a two-node power system, which is cleared by using uniform pricing settlements. Nanduri and Das (2007) assess the market power of generation companies under auction-based pricing for a day-ahead energy market. 


\section{Zhou, Chan, Chow and Kotsan}

Although potential collusive behavior has been reported in wholesale electricity pool markets in, for example, Spain (Fabra and Toro 2005), California (Puller 2007), and England and Wales (Bunn and Martoccia 2005), related studies focusing on collusive behavior of generators in repeated games are limited. Two of such studies are by Cau (2003) and Cau and Anderson (2003). In Cau (2003), two identical generators supply electricity at the same node, hence no network congestion is modeled. The author applies a co-evolutionary genetic algorithm to the generator agents to allow them to bid strategy over time. It is shown that collusive behavior resulting from learning may occur in a stylized electricity market even the system does not converge to a stable equilibrium outcome. This model assumes that the number of players and their strategy spaces are public information. With similar market settings, Cau and Anderson (2003) study the tacit collusion of generators in oligopolistic electricity markets in which generators' bidding structure is piecewise linear.

In this paper, we set up an ABS simulation model for a five-node electric power system with customized transmission congestion constraints and real time elastic demand. We apply an online-hill climbing learning algorithm to generator agents who have market power to bid strategically but with limited information about the market, including information of the opponents. The generator agents, however, can set their goals of market share and bid strategically to reach their goals. The objectives of this study are to examine what bidding behavior (collusive or competitive) will result under these market settings with accurate or inaccurate market goals and under the online-hill climbing learning algorithm.

\section{AN ABS MODEL OF AN ELECTRICITY MARKET}

In this section, we present an agent-based simulation model for a five-node electricity market including generation companies, load serving entities (LSEs), transmission companies, and ISO.

\subsection{Objective}

During the course of a repeated bidding game, what a generation company knows are its own historical bids, winning quantities, and clearing prices (if the market is cleared by the discriminatory pricing mechanism, generation companies only know their own winning prices). The generation company do not know their opponents bidding strategies and winning quantities. Nevertheless, this limited information is critical for the generation companies to make decisions on the next bid. Therefore, to make rational decisions, generation companies must have the ability to learn to estimate the bidding strategies of their opponents. The objective of our study is to understand the bidding behavior of generation companies under the assumption that the generation companies can accurately estimate their market share.

\subsection{Physical Model}

To make the simulation close to real world model and reveal nontrivial result, we set up a stylized electricity market with comprehensive characteristics of both physical transmission network and market configuration.

\subsubsection{Transmission network}

The ABS model considered in this paper is based on a five-zone transmission network. The topology of the power system is illustrated in Figure 1. Each zone consists of one LSE and up to ten generators. These zones are inter-connected through six transmission lines with capacity limit. While the simulation model is designed to allow active power flow constraints on any of the lines, in this paper we only consider the constraint that the total amount of active power transmitted from Zones 3 and 2 to Zone 4 is restricted to $T_{4}$, an adjustable parameter.

\subsubsection{Participants}

In the restructuring process, a power system is separated into generation, transmission, distribution, and system operator, which are the major participants in an electricity market. In our simulation model, they are called generation companies, transmission companies, LSEs, and ISO, respectively. The LSEs are the customers in a wholesale market. They contract with other market participants to purchase electricity to satisfy the demand in their corresponding service areas. The generation companies are the suppliers in the electricity market. They compete with each other to sell their electricity through an auction market or bilateral contracts. The transmission companies are organizations that own, maintain, and operate transmission assets for profit subject to regulation. The ISO is an organization that coordinates, controls, and monitors the operations of the electric power system. Some ISOs also act as the regulators in their electricity markets, including the wholesale markets. 


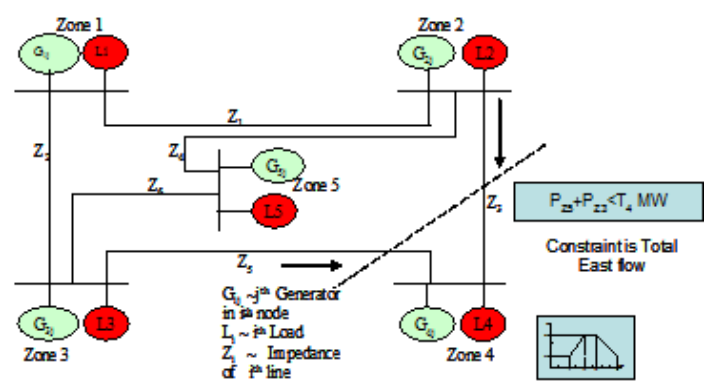

Figure 1: A Five-Zone Power System

\subsubsection{Auction Procedure}

In the day-ahead auction market of our simulation model at day $t$, the LSEs estimate the load demand in their service areas for each hour of the next day (day $t+1$ ) and submit that information to the ISO as load bid. The generation companies decide their bids by using their knowledge about the environment and competitors. Before the end of day $t$, the ISO closes the market for day $t+1$ and clears the market. By selecting the cheapest electricity with a set of constraints including physical limits, the ISO calculates the market clearing price using a uniform pricing mechanism. Meanwhile, the ISO decides the generation and distribution scheduling information and return them to the generation companies and LSE. On day $t+1$, the generation companies schedule their operations and generate the amount of electricity they were committed to on day $t$. The LSEs receive the amount of electricity based on their bids and distribute it to their end customers.

\subsection{Agents}

Our model implements the three types of agents correspond to the three basic participants in the electricity market: generation company agents, LSE agents, and ISO agent.

\subsubsection{Generator agents}

Each generation company, without loss of generator, is assumed to own only one generator, represented as a generator agent. Generator agents are differentiated by their maximum generation capacities and real cost functions.

\section{Generator capacity, cost function}

Generation capacity here means the maximum output of a generator. A cost function describes the relationship between the output quantity and production cost. Basically, it includes the fixed cost, variable cost, and cost for starting up and shutting down the machines if they are used. For simplicity, only the variable cost is considered in the following. The cost function used in this model is a polynomial cost function, which is defined in the following equation

$$
C_{j}\left(q_{j}\right)=c_{j 0} q_{j}^{2}+b_{j 0} q_{j}+a_{j 0}, q_{j} \in\left[0, M O_{j}\right],
$$

where $M O_{j}$ is the maximum capacity, $q_{j}$ is the amount of electricity supplied by generator $G_{j}, C_{j}\left(q_{j}\right)$ is the cost for $G_{j}$ to supply $q_{j}$ units of electricity, and $c_{j 0}, b_{j 0}$, and $a_{j 0}$ are the coefficients of the cost function.

2. Bidding strategies

When a generator agent submits its bid to the ISO, it is required to report its supply function. If the agent reports its real cost function as supply function, it is called a marginal cost bidder (MCB). Another situation is that it can report an adjusted cost function as its supply function. In this case, the agent tries to be profit. There are two ways to adjust a bid: capacity withholding and price adjustment. Figure 2 gives an example for price adjustment. In the figure, the agent can report a cost function (dotted line) higher than its real cost function (solid line). If the agent has market power, its objective could be maximizing its profit from this adjustment.

Because the price is decided by the supply function, price adjustment is implemented by raising or lowering the coefficients of the supply function. The adjustment is represented by the markup rate compared to the coefficients 
Zhou, Chan, Chow and Kotsan

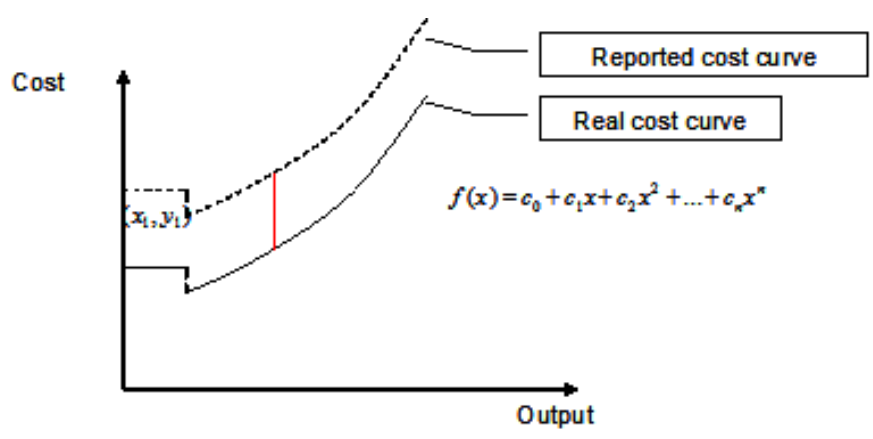

Figure 2: Supplier bids above its marginal cost function

of the marginal cost function. For example, if a generation company bids at a price $150 \%$ higher than its marginal cost, its markup rate is 2.5 . If it bids at its marginal cost, its markup rate is 1 .

Because bidding in an electricity pool market is a repeated game, generators can learn from their bidding history and market environment to improve their profit. In our model, the agents can learn by using an online hill climbing algorithm with adjustment (OHCA), which is introduced in Section 3.4.

\subsubsection{LSE}

There is one LSE agent in each zone. In our simulation model, its only task is to forecast the load demand for the next day and report it to the ISO agent. The demand reported by the LSE agents can be elastic both time and price (see in Section 3.4 for details). The LSE agents do not take strategic actions. Their costs, however, are minimized with the help of the ISO by selecting the cheapest generators. It should be noted that the parameters of the demand responsive curve are constant during the simulation.

\subsubsection{ISO}

The objective of the ISO is to regulate the market by maximizing the social welfare or minimizing the total cost to meet the load demand. The ISO agent selects the lowest cost generators with a higher priority. The constraints that prevent the ISO agent from selecting all lowest cost generators include the generators' capacity, transmission capacity, and power system reliability.

The ISO agent collects bids from the generator and LSE agents before the market is closed. Then, it clears the market by solving an optimal power flow problem. After the market is cleared, the ISO agent informs the generator and LSE agents their scheduling information. If the market is cleared with a uniform price, then this price is open to the public. Otherwise, it is private, i.e., only sent to the related agents.

\subsection{Experiment Settings}

The following are the settings and assumptions on the market structure and physical transmission system of our model.

\section{Generation capacity and demand distribution}

There is one generator agent in each zone except in Zone 4 (see Figure 1). In Zone 4, there are six generators, denoted by $G_{j}(j=1, \ldots, 6)$. Compared to the generators in Zone 4, other generators have cheaper production costs and can supply the demand in Zone 4 after satisfying their own demand. However, the amount of electricity exported to Zone 4 is limited by the transmission line capacity, which is denoted by $T_{4}$. Among the six generators in Zone 4, two of them (i.e., $G_{1}$ and $G_{2}$ ) are identical companies with generation capacity larger than the sum of the other four small generators, who are also identical. These four smaller generators are price-takers; they do not bid strategically. The two large generators can use different pricing strategies to be more profit. To facilitate our study, the total amount of generation supply in the market is assumed to be greater than the total demand even under transmission congestion. 

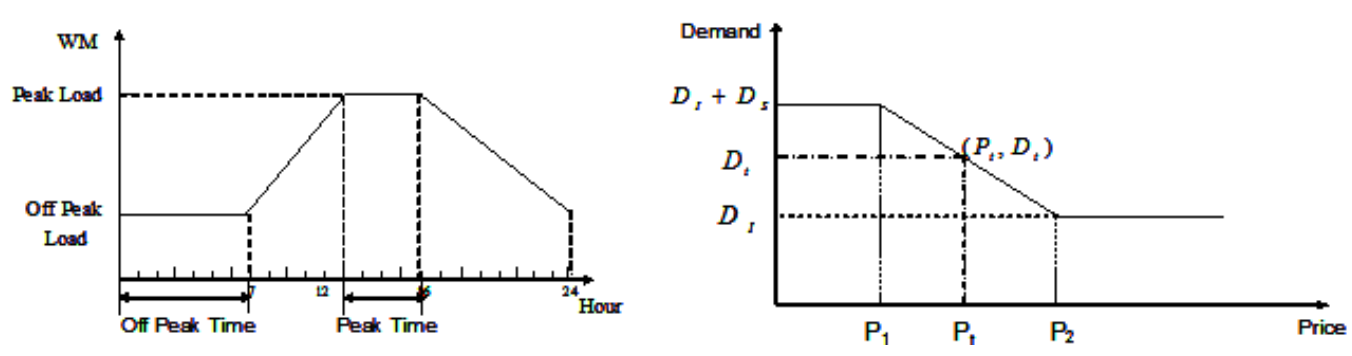

Figure 3: The Characteristics of the Demand

The demand has the two characteristics: First, it varies during a 24-hour period as illustrated in the left part of Figure 3 with peak hours and off-peak hours in the middle of the day and early morning, respectively. Figure 3 shows the demand curve for one day. Second, the demand is a price-responsive curve (the right part of Figure 3) for each hour of next day to the ISO.

The submitted load demand is divided into two parts: inelastic demand $\left(D_{I}\right)$ and elastic demand $\left(D_{S}\right)$. The elastic part drops when the price increases and reaches zero if the price is too high. This elasticity is negatively proportional to the price, as shown in the right part of Figure 3. In this part, the y-axis is the demand during the peak time. The x-axis is the price. When the price is less than $P_{1}$ (the lowest price at which the elastic part of the load demands begins to drop), there is no decrease in the elastic load demand. When the price is greater than $P_{1}$ but less than $P_{2}$, the elastic part begins to decrease proportional to the price. When the price is greater than $P_{2}$ (the lowest price at which the elastic load demand drops to zero), the demand remains at $D_{I}$ because only the inelastic load demand is left.

2. Market settings

The market is cleared by the ISO using locational-based marginal price (LBMP), which means that the price is uniform within each zone and different among zones due to the transmission congestion.

3. Market share

In this experiment, generator agents are assumed to be able to estimate their market share. Because the two generators are identical, they could share equally the market. They take this information into consideration when they bid.

4. Learning algorithm for generation company agents

The online hill climbing with adjustment (OHCA) algorithm is similar to those used in optimal search procedures. The intuition of the OHCA algorithm is that an agent increases its bidding price if it becomes more profitable and decreases otherwise. In this paper, we set the amount of adjustment based on the agent's targeted market share. The detailed description of the OHCA used by generation company agents is provided in the following subsection:

\section{(a) Notation}

The notation used in this section are listed in Table 1.

Table 1: Notation for OHCA algorithm

\begin{tabular}{|c|l|}
\hline Symbol & \multicolumn{1}{|c|}{ Meaning } \\
\hline$\beta_{t i j}$ & Adjustment rate decided by the difference between targeted market share and current market share \\
\hline$b_{j 0}$ & First order coefficient of the marginal cost function for the $j^{t h}$ generator \\
\hline$c_{j 0}$ & Second order coefficient of the marginal cost function for the $j^{\text {th }}$ generator \\
\hline$c_{t i j}$ & Coefficient of the cost function in round $t$ for the $i^{t h}$ hour reported to the ISO by the $j^{t h}$ generator \\
\hline$d_{t i j}$ & Adjustment rate decided by the change of market share \\
\hline$G_{j}$ & The $j^{t h}$ generator agent \\
\hline$P_{t i j}$ & Profit in round $t$ for the $i^{t h}$ hour for the $j^{t h}$ generator \\
\hline$r_{t+1, i, j}$ & Change rate on the coefficients of cost function in round $t+1$ for the $i^{t h}$ hour for the $j^{\text {th }}$ generator \\
\hline$S_{j 0}$ & Targeted market share for the $j^{t h}$ generator \\
\hline$s_{t i j}$ & Market share in round $t$ for the $i^{t h}$ hour for the $j^{t h}$ generator \\
\hline$\tau_{0}$ & A constant multiplier to adjust the step size of markup rate \\
\hline
\end{tabular}




\section{Zhou, Chan, Chow and Kotsan}

(b)

\section{Action and Adjustment}

We are mainly interested in the bidding behavior of the two large generators at Zone 4 because all the other generators are marginal bidders and do not employ bidding strategies. As introduced earlier, these two generators bid strategically by updating their bids. They change the bids by adjusting the mark-up rate of the first two coefficients of the cost functions. In particular, the first and second coefficients (i.e., $c_{t+1, i, j}$ and $b_{t+1, i, j}$ ) of the reported cost function of Generator $j$ (i.e., $\left.G_{j}\right)$ at the $(t+1)^{t h}$ round are adjusted by the following equations:

$$
c_{t+1, i, j}=c_{t i j}+c_{j 0} r_{t+1, i, j} / \tau_{0}
$$

and

$$
b_{t+1, i, j}=b_{t i j}+b_{j 0} r_{t+1, i, j} / \tau_{0}
$$

where $r_{t+1, i, j}$ is the bidding markup rate, which controls the amount of adjustment on both coefficients of the reported cost function in a bid. The constant multiplier, $\tau_{0}$, is used here to manage the magnitude of the step size. Its value might affect the convergence time or stability of the equilibrium. In all the experiments in the following, a value of 8 is used and a reasonable convergence time and stability is obtained. In this paper, we consider three factors that could influence this adjustment: (a) the change in the profit, (b) the change in the market share, and (c) the distance to the targeted market share. The first two factors together result in four possible cases: (1) profit increases and market share increases, (2) profit increases but market share decreases, (3) profit decreases but market share increases, and (4) profit decreases and market share decreases. Gaining more profit is a high priority; therefore, the amount of adjustment should be positive in both Cases (1) and (2) (i.e., increase the bid to gain more profit) and negative in both Cases (3) and (4) (i.e., decrease the bid to retain the profit). The magnitude of the adjustment in these four cases is determined by the amount of changes in the market share in the unit of the targeted market share. In summary, the first two factors (i.e., Cases (1) - (4)) contribute to the adjustment of markup rate in the format of the following equations:

$$
d_{t+1, i, j}=\left\{\begin{array}{lll}
\frac{s_{t-1, i, j}-s_{t i j}}{S_{j 0}} & \text { if } & P_{t i j} \geq P_{t-1, i, j} \text { and } s_{t-1, i, j}>s_{t i j} \\
1 & \text { if } & P_{t i j} \geq P_{t-1, i, j} \text { and } s_{t-1, i, j} \leq s_{t i j} \\
-1-\frac{s_{t-1, i, j}-s_{t i j}}{S_{j 0}} & \text { if } & P_{t i j}<P_{t-1, i, j} \text { and } s_{t-1, i, j}>s_{t i j} \\
-1 & \text { if } & P_{t i j}<P_{t-1, i, j} \text { and } s_{t-1, i, j} \leq s_{t i j}
\end{array}\right.
$$

The last factor contributing to the adjustment is the distance between the current market share and the targeted market share. Specifically, if the current market share is less than the targeted market share, $G_{j}$ takes a conservative action by multiplying the adjustment with a factor equal to the difference between the targeted market share and the current market share in the unit of the targeted market share. If $G_{j}$ gets more market share than it expects, it has no incentive to make adjustment and therefore, this factor is one.

$$
\beta_{t+1, i, j}= \begin{cases}\frac{S_{j 0}-s_{t i j}}{S_{j 0}} & \text { if } \quad s_{t i j}<S_{j 0} \\ 1 & \text { otherwise }\end{cases}
$$

Eq. 4 and Eq. 5 together determine the exact amount of adjustment in the markup rate, $r_{t+1, i, j}$, based on the following equation:

$$
r_{t+1, i, j}=d_{t+1, i, j} b_{t+1, i, j}
$$

\section{(c) Termination criteria}

The simulation is terminated when either the maximum number of iterations (simulation days) is reached or there is no more profit gain in all the generator agents. The second stopping condition means that an equilibrium is obtained given the set of strategies used by the generator agents. 


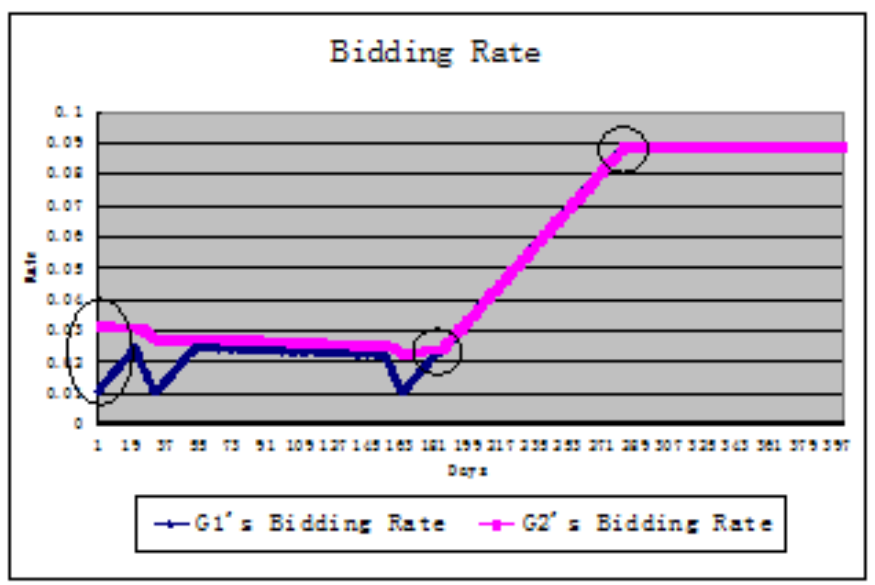

Figure 4: G1 and G2 Bidding Rates VS Time

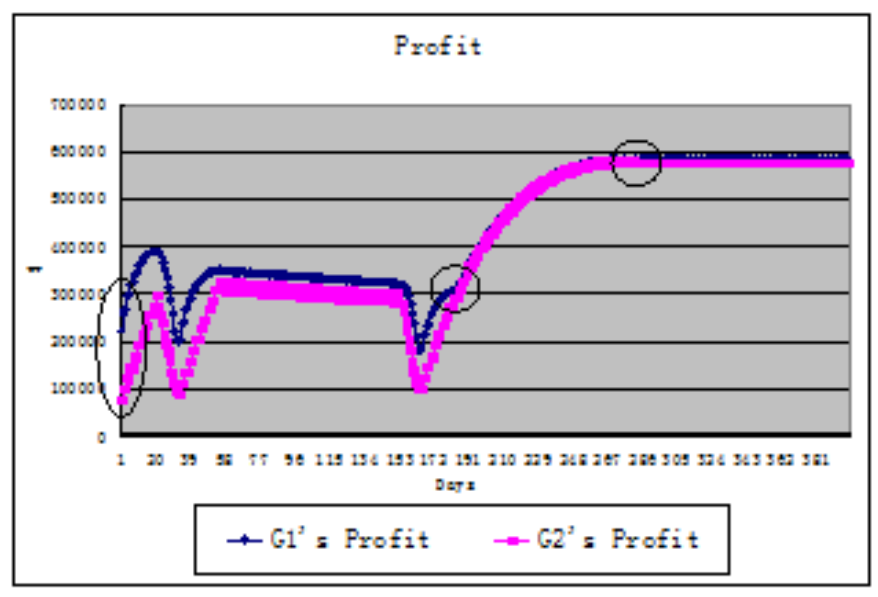

Figure 5: G1 and G2 Profits VS Time

\section{SIMULATION RESULTS}

Before discussing the simulation results, it should be pointed out that the other four smaller generators and the generators outside Zone 4 are cheaper generators than $G_{1}$ and $G_{2}$. As a result, $G_{1}$ and $G_{2}$ are only competing for the total demand less those satisfied by all the other generators, which will always be picked by the ISO.

We study two types of market scenarios: accurate market goals and inaccurate market goals. In the accurate case, because the two generators are identical, their expected market shares are each set to 50\%. In the inaccurate case, both generators overestimate their market power and set each of their market shares to $60 \%$. In all the simulations below, the maximum number of iterations is set to 2500 (days or rounds). All simulations are run on a $2.5 \mathrm{GHZ}$ dual-core PC with 3 GB of RAM.

\subsection{Accurate Targeted Market Share}

Figures 4, 5, and the left part of Figure 6 show the dynamics of the supply bids, profits, and clearing prices, respectively, under the scenario of accurate market goals. The initial markup rates on the marginal cost of the two generators are 1 and 3 , respectively. This means that $G_{1}$ bids at its marginal cost while $G_{2}$ bids 2 times higher than its marginal cost. 

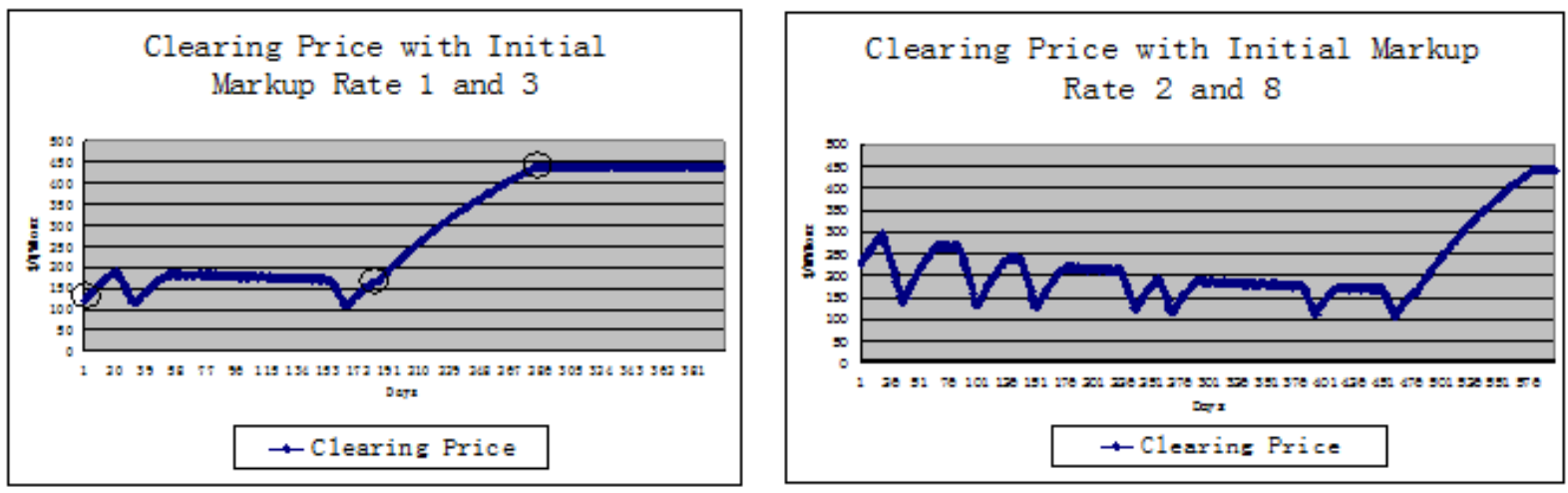

Figure 6: Market Clearing Price VS Time with Different Initial Markup Rates
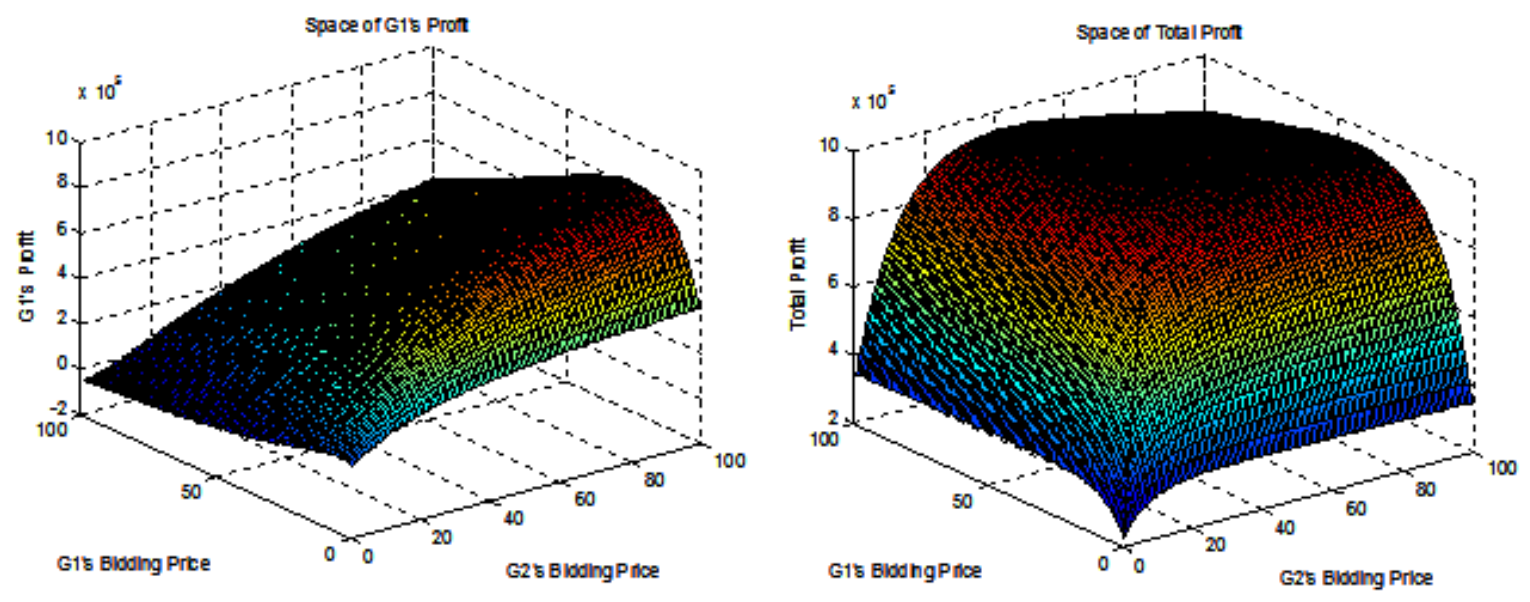

Figure 7: Surface Plots of the G1 and G2 Profits

Figure 5 shows three important points, which are encircled. The first one is the initial point where the profit of $G_{1}$ is greater than that of $G_{2}$ because $G_{2}$ 's supply is lower. Correspondingly, $G_{1}$ 's market share is higher than that of $G_{2}$. As the two generators start to learn and adjust their strategies, their profits evolve to equality (because they are identical). This first occurs at the second encircled point in Figure 5. After the equality point (i.e., equal market share) is reached, the two generators behave like a coalition but they in fact bid individually, independently, and identically to maximize their profits individually because they do not communicate with each other. The maximum point happens at the maximum price just before the demand starts to drop (at the third circle in Figure 5).

Two transition periods are worthy of discussion here: The first one is the period during which the two generators learn to reach equality (from point 1 to point 2) and the second one is the period during which the two generators learn to reach maximum (from point 2 to point 3 ). We first enumerate $G_{1}$ 's profit based on its bidding price and $G_{2}$ 's bidding price (see the left part of Figure 7). One can see from the left part of Figure 7 that for a fixed bidding price of $G_{1}, G_{1}$ 's profit increases in $G_{2}$ 's bidding price. On the contrary, for a fixed bidding price of $G_{2}, G_{1}$ 's profit decreases in its bidding price.

The right part of Figure 7 shows the sum of the profits of the two generators with respect to their supply bids. The arrow in the center of the figure illustrates the collection of points where the two generators equally share the market.

Because the demand is responsive to the market clearing price as illustrated in the right part of Figure 3, there is a point where the LSE payment is maximized. Moreover, because the payment of the LSEs is related to the profit of generators, there should be a point where the total profit of all generators is maximized. This can be considered as a multi-player Nash equilibrium. If the generators in the market have the intension to collude implicitly or explicitly even though they do not communicate with each other, they might try to explore this maximum point and stay there given that they are identical 


\section{Zhou, Chan, Chow and Kotsan}

and understand that they cannot beat each other by deviating from the collusion. Moreover, because the two generators are identical, they share the market equally in steady state. Otherwise, the market is not in steady state and one of generators can change its bidding strategy to gain more market share and profit.

Therefore, regardless of what their initial supply bids are, they learn to find the point where they share the market equally (the first transition period), which occurs at some point on the line indicated by the arrow in the right part of Figure 7. Because there is a maximum on that line, the two generators will bid strategically to reach that maximum.

We test different combinations of initial bidding prices as shown in Table 2. Because $G_{1}$ and $G_{2}$ are identical, we only use the bidding rate of $G_{1}$ of 1 and 2 (the $1^{\text {st }}$ column) and the bidding rate of $G_{2}$ from 1 to 9 with unity increment (the $1^{\text {st }}$ row). Each entry in Table 2 gives the clearing price under the corresponding combination of the bidding rates of $G_{1}$ and $G_{2}$. All experiments result in a similar clearing price (equilibrium). Only the transition periods are different. Therefore, the experimental results show that the OHCA learning algorithm under the market settings in this paper can lead to a collusive behavior of the two generators and that the two generators can always reach the market equilibrium at which their total profit is maximized regardless of what the initial bidding rates are.

Table 2: Market Clearing Price under Different Initial Bidding Price

\begin{tabular}{c|rrrrrrrrrr}
\hline \multirow{2}{*}{ Bidding Rate of $G_{1}$} & \multicolumn{11}{c}{ Bidding Rate of $G_{2}$} \\
& & & & & & & & & & \\
& 1 & 2 & 3 & 4 & 5 & 6 & 7 & 8 & 9 \\
\hline 1 & 429.66 & 435.85 & 438.24 & 438.54 & 440.16 & 439.91 & 436.72 & 436.59 & 439.28 \\
\hline 2 & 435.85 & 435.93 & 436.5 & 440.24 & 439.28 & 436.96 & 437.36 & 440.02 & 439.94 \\
\hline
\end{tabular}

The initial bidding rates, however, do affect the time to reach the equilibrium. The right part of Figure 6 shows the dynamics of the changes in the clearing price when the initial markup rates are 2 and 8 for $G_{1}$ and $G_{2}$, respectively. The time for the two generators to reach the equality point is much longer than the case we showed in the left part of Figure 6 because the difference between the initial bidding rates of $G_{1}$ and $G_{2}$ in this case is larger than that in the former case, which makes these two generators spent more time to learn to evolve to equality.

\subsection{Biased Targeted Market Share}

Equally sharing a market is an ideal result. In practice, however, generators are usually competing for the market share based on their estimated market power. The problem is that they might not have an accurate estimate of their market power. As a result, they might set a wrong targeted market share. The consequence could be a price war, which is the case observed in the experiments below.

This section tries to answer the question "what would happen if both generators expect more than $50 \%$ of the market share?" In particular, we experiment with the scenario where both $G_{1}$ and $G_{2}$ expect $60 \%$ of the market share with everything else being equal. The initial bidding rates for $G_{1}$ and $G_{2}$ are 1 and 3, respectively.

Figure 8 illustrates the dynamics of the bidding rates and profits of the two generators. It can be seen that the bidding rates of both generators converge to the marginal rate (the left part of Figure 8) and the profits reduce to the one obtained at the marginal cost for both generators (the right part of Figure 8). This result implies that under the OHCA algorithm, if both generators erroneously or maliciously set a targeted market share larger than what their market power can achieve, they will end up in a price war.

\section{CONCLUSION}

In this paper, we present an agent-based simulation model on a stylized electricity pool market. We apply the online hill climbing with adjustment (OHCA) algorithm to generator agents to guide them to maximize their profits. When the agents accurately (or genially) set their targeted market shares equal to their market power, collusive bidding behavior is observed and equilibrium is obtained when the total profits of the agents are maximized. However, when the agents erroneously (or maliciously) set their targeted market shares greater than their market power, the consequence will be a price war and their profits will be minimized (rather than maximized) to be equal to the marginal-cost profit.

The main contributions of this paper are the following. 

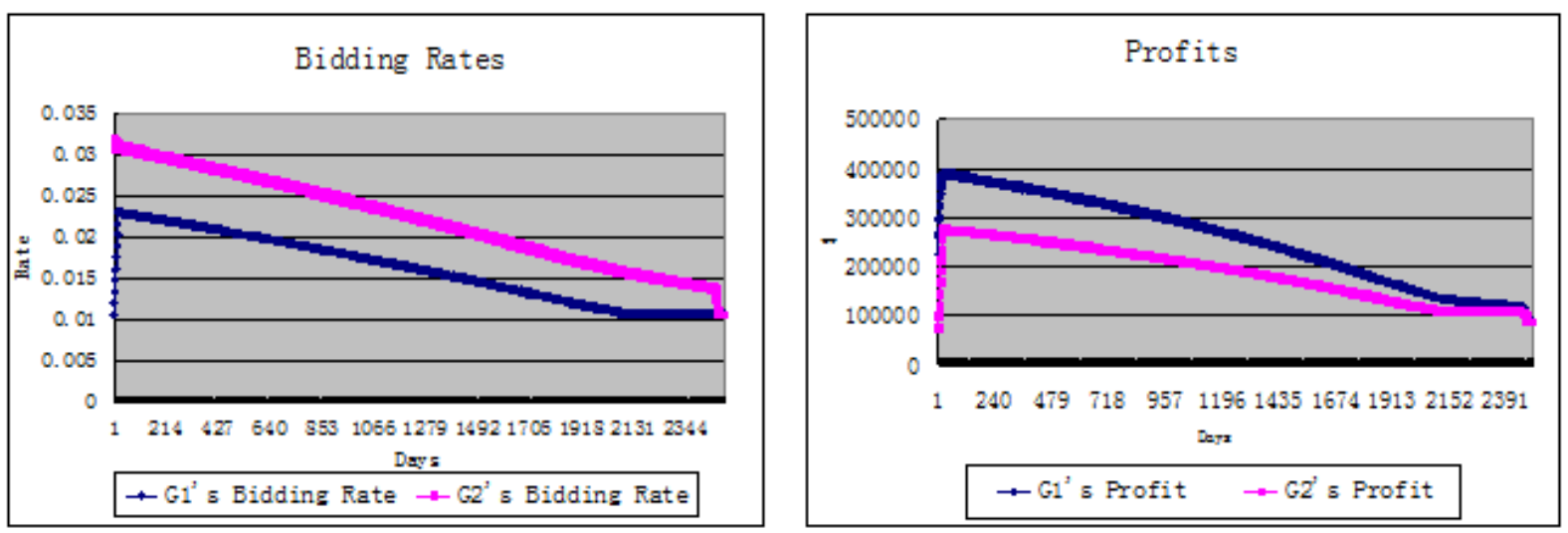

Figure 8: The Dynamics of the Bidding Rates and Profits with Biased Targeted Market Share

1. This paper presents an agent-based simulation model with several characteristics, including active power flows limits and real-time elastic demand.

2. It is shown that under the OHCA algorithm, collusive behavior (resulting from non-communicated cooperation through learning) can maximize profits while competing behavior (resulting from inaccurate market goals) will minimize profits.

3. The experimental results show that with an accurate targeted market share, the agents' profits increase simultaneously after they reach the equality point and that the first maximum point after equality is always the global profit maximum point. This finding not only gives insights about the effect of bidding behavior of two identical generators when they try to reach their targeted market share, but also provides an indication of how two independent (with no communication) generators maximize their total profit using a relatively simple OHCA algorithm.

4. The experimental results also imply that with an inaccurate targeted market share, the agents' profits will be equal to the marginal-cost profit, that is, a price war. This result is consistent with the Bertrand games (Kreps and Scheinkman 1983).

Real-world generators are much more complex. Therefore, future work includes testing asymmetric generators and simulating different learning algorithms with inaccurate estimation of the market share.

\section{ACKNOWLEDGMENTS}

The authors wish to thank the NYISO for supporting the research reported here.

\section{REFERENCES}

Borenstein, S., and J. Bushnell. 1999. An empirical analysis of the potential for market power in california's electricity industry. The Journal of Industrial Economics 47.

Bower, J., and D. Bunn. 2001. Experimental analysis of the efficiency of uniform-price versus discriminatory auctions in the england and wales electricity market. Journal of Economic Dynamics and Control 25:561-592.

Bunn, D., and M. Martoccia. 2005. Unilateral and collusive market power in the electricity pool of england and wales. Energy Economics 27:305-315.

Bunn, D., and F. Oliveira. 2001. Agent-based simulation-an application to the new electricity trading arrangements of england and wales. IEEE Transactions on Evolutionary Computation 5.

Cau, T. 2003. Analyzing tacit collusion in oligopolistic electricity markets using a co-evolutionary approach. Ph.D. thesis, Australian Graduate School of Management, University of New South Wales and University of Sydney.

Cau, T., and E. Anderson. 2003. A co-evolutionary approach to the tacit collusion of generators in oligopolistic electricity markets: piecewise linear bidding structure case. The 2003 Congress on Evolutionary computation 4:2306-2313.

Fabra, N., and J. Toro. 2005. Price wars and collusion in the spanish electricity market. International Journal of Industrial Organization 23:155-181. 


\section{Zhou, Chan, Chow and Kotsan}

FERC 2006. United states of america electricity energy market competition task force and the federal energy regulatory commission, government report. FERC.

Friedman, D., and J. Rust. 1993. The double auction market: Institutions, theories, and evidence. Westview Press.

Green, R., and D. Newbery. 1992. Competition in the british electricity spot market. Journal of Political Economy 100.

Klemperer, P., and M. Meyer. 1989. Supply function equilibria in oligopoly under uncertainty. Econometria 57:1243-1277.

Kreps, D., and J. Scheinkman. 1983. Quantity precommitment and bertrand competition yield cournot outcomes. The Bell Journal of Economics 14:326-337.

Micola, A., A. Banal-Estanol, and D. Bunn. 2008. Incentives and coordination in vertically related energy markets. Journal of Economic Behavior \& Organization 67:381-393.

Nanduri, V., and T. Das. 2007. A reinforcement learning model to assess market power under auction-based energy pricing. IEEE Transactions on Power Systems 22:85-95.

Nicolaisen, J., V. Petrov, and L. Tesfatsion. 2001. Market power and efficiency in a computational electricity market with discriminatory double-auction pricing. IEEE Transactions on Evolutionary Computation 5.

Puller, S. 2007. Pricing and firm conduct in california's deregulated electricity market. The Review of Economics and Statistics 89:75-87.

Rudkevich, A., M. Duckworth, and R. Rosen. 1998. Modeling electricity pricing in a deregulated generation industry: The potential for oligopoly pricing in a poolco. Energy Journal 19:19-48.

Tellidou, A., and A. Bakirtzis. 2007. Agent-based analysis of capacity withholding and tacit collusion in electricity markets. IEEE Transactions on Power Systems 22.

von der Fehr, N., and D. Harbord. 1993. Spot market competition in the uk electricity industry. The Economic Journal 103:531546.

Wu, Q., and J. Guo. 2004. Optimal bidding strategies in electricity markets using reinforcement learning. Electric Power Components and Systems 32:175-192.

\section{AUTHOR BIOGRAPHIES}

ZHI ZHOU is a PhD student in the department of Decision Science and Engineering System at Rensselaer Polytechnic Institute. He received his bachelor's degree and master's degree in computer science from Wuhan University, China, in 2001 and 2004, respectively. His research interests are simulation, multi-agent systems, and electricity markets. His email address is <zhouzerpi.edus.

WAI KIN VICTOR CHAN is an Assistant Professor of the Department of Decision Sciences and Engineering Systems at Rensselaer Polytechnic Institute. He received his bachelor's degree and a master's degree in electrical engineering from, respectively, Shanghai Jiao Tong University, China in 1997, and Tsinghua University, China in 2000, and M.S. and Ph.D. degrees in industrial engineering and operations research from University of California, Berkeley in 2001 and 2005, respectively. His research interests include discrete-event simulation, agent-based simulation, mathematical programming, and their applications in energy markets, social networks, service systems, transportation networks, and manufacturing. His e-mail address is <chanwerpi.edu $>$.

JOE H. CHOW is a Professor of Electrical, Computer, and Systems Engineering and Associate Dean of Engineering for Research and Graduate Programs at Rensselaer Polytechnic Institute, Troy, New York. He received his BS degrees in Electrical Engineering and Mathematics from the University of Minnesota, and the MS and PhD degrees from the University of Illinois, Urbana-Champaign. He worked at General Electric Company before joining RPI in 1987. His current research includes Voltage-Sourced Converter based Flexible AC Transmission Systems, and the analysis, modeling, and control of large power systems using synchronized phasor measurements. He has received several awards in control and power systems, and is a fellow of IEEE.

SERHIY KOTSAN is an Economist of the Market Monitoring Unit at the New York Independent System Operator. He received his bachelor's degree in Financial Management from Hogeschool van Utrecht in 1998, and M.A. and Ph.D. degrees in economics from West Virginia University in 2003 and 2005, respectively. His research interests include game theory, industrial organization and their application in electricity markets. His e-mail address is <skotsan@nyiso.com>. 